\title{
Reflection of electromagnetic waves from mixtures of plane gravitational and scalar waves
}

\author{
O. Gurtug, ${ }^{*}$ M. Halilsoy, ${ }^{\dagger}$ and O. Unver ${ }^{\ddagger}$ \\ Department of Physics, Eastern Mediterranean University, G. Magusa, north Cyprus, via Mersin 10, Turkey \\ (Received 4 January 2006; published 17 August 2006)
}

\begin{abstract}
We consider colliding wave packets consisting of hybrid mixtures of electromagnetic, gravitational, and scalar waves. Irrespective of the scalar field, the electromagnetic wave still reflects from the gravitational wave. Some reflection processes are given for different choice of packets in which the Coulomb-like component $\Psi_{2}$ vanishes. Exact solution for multiple reflection of an electromagnetic wave from successive impulsive gravitational waves is obtained in a closed form. It is shown that a successive sign flip in the Maxwell spinor arises as a result of encountering with an impulsive train (i.e. the Dirac's comb curvature) of gravitational waves. Such an observable effect may be helpful in the detection of gravitational wave bursts.
\end{abstract}

DOI: 10.1103/PhysRevD.74.044020

PACS numbers: 04.20.Jb

\section{INTRODUCTION}

Reflection of electromagnetic (em) waves in general relativity is totally different from its counterpart process of classical electromagnetism. While in the latter the incident em wave is partly reflected and transmitted, the reflector remains unaffected. Corresponding process in general relativity is a completely different process, as a manifestation of inherent nonlinearity the reflector wave pulse is modified as well. Thus exact solution of the full Einstein-Maxwell (EM) equations respecting all boundary conditions becomes indispensable.

The problem of colliding em waves with gravitational waves has not yet been solved in the general case but only, in particular, situations. The solution by Griffiths corresponds to the most elementary one [1-3]. Closely related to the subject are the solutions of colliding impulsive lightlike signals [4] and the lightlike shells with impulsive gravitational waves in scalar-tensor theory of gravity [5]. The problem of reflection is interesting in the sense that it incorporates a nonsymmetrical initial data in the collision process. The exact analytic solution is obtained with an imposed condition that one of the metric functions characterizing the gravitational degrees of freedom depend only on one of the null coordinates as it was in the precollision region. Significant reduction in the field equations follows as a result of this condition.

In Griffiths's work, exact solutions for two different cases were given; one corresponding to an impulsive, the other to a shock gravitational wave. The main feature of the problem is that the em wave partly reflects from the gravitational wave while the latter does not reflect from the em wave. As a result of the nonlinear interaction a Coulomb-like (i.e. $\Psi_{2}$ in the Newman-Penrose formalism) component develops apart from the modified wave component $\Psi_{4}$. Another feature in this process is that in the

\footnotetext{
*Electronic address: ozay.gurtug@emu.edu.tr

Electronic address: mustafa.halilsoy@emu.edu.tr

Electronic address: ozlem.unver@emu.edu.tr
}

interaction region a spacelike curvature singularity necessarily develops.

The purpose of this paper is to present new extensions to the same physical process by employing the solution of Griffiths. One of the extensions is to consider the collision and interaction of plane gravitational radiation and em waves in the presence of a massless scalar field. Another extension is to consider the nonlinear interaction of a plane em wave with the succesion of plane impulsive gravitational waves.

The main motivation to consider the scalar field in this problem is that, in recent years there is a growing literature around the subject of scalar field cosmology. With this in mind, it would be physically interesting to investigate the effect of scalar field taking part in the reflection problem of em waves. In addition to this, we are aiming to find a nonsingular Cauchy-horizon $(\mathrm{CH})$ forming reflection process. To incorporate scalar fields in this direction, we construct $\mathrm{CH}$ forming Einstein-scalar and em-scalar plane wave packets that propagate in the opposite directions in each of the incoming regions as shown in Fig. 1. The resulting space-time is as a result of the collision of these wave packets.

The gravitational wave in our problem has a different structure compared to the cases considered in Refs. [1-3]. In the problem considered, the gravitational radiation is composed of a plane impulsive part accompanied with shock waves. The obtained solution has revealed in contrast to our expectation that, it does not yield a $\mathrm{CH}$ forming reflection process. Our solution implies that the em wave still partly reflects from the gravitational wave irrespective of the presence of the scalar field. Following the collision, the resulting space-time has the $\Psi_{4}$ and $\Psi_{2}$ components and they are both unbounded as the focusing hypersurface is approached. General feature of the problem has not been changed with the insertion of the scalar field, but we have shown that a particular choice of the scalar field makes $\Psi_{2}=0$, with the only nonzero component $\Psi_{4}$. This provides a unique example in which the scalar field can rule the gravitational field. 


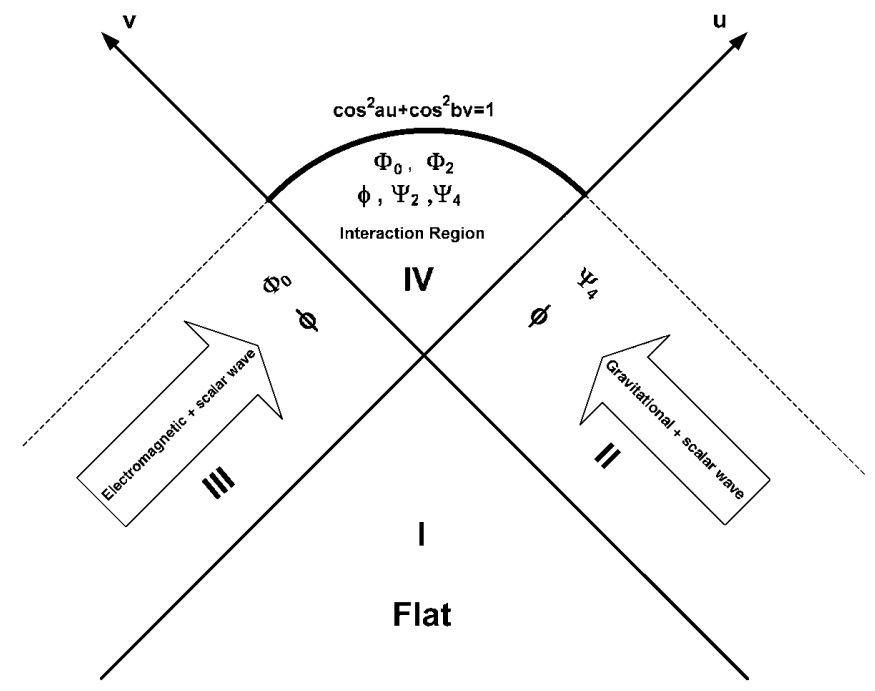

FIG. 1. The space-time diagram describes the collision of wave packets that contains em + scalar waves in one of the incoming region and gravitational + scalar waves in the other incoming region. In this process the em wave still partly reflects.

Another extension is naturally provided by considering an em wave that encounters a train of impulsive gravitational waves (i.e. the Dirac's comb). The solution describes simply, how the em wave reflects from the successive gravitational impulsive waves. In principle the problem is one pertaining to multiple reflection but idealized choice of gravitational impulsive wave train renders an exact solution possible. We show that the result is similar to the single impulsive wave case, with the additional property that the reflecting Maxwell component changes sign while crossing from one wave to the next. This fact may play role in the identification of succesive impulsive gravitational waves which remain still elusive to any detection technique. The whole discussion can also be extended to a gravitational wave with nonaligned polarization, however for convenience reasons we shall restrict ourselves in this paper to the linear polarization.

Organization of the paper is as follows: In Sec. II, we construct $\mathrm{CH}$ forming Einstein-scalar and em-scalar plane wave spacetimes. In Sec. III, we consider the collision and subsequent interaction of these wave packets. A particular scalar field that makes $\Psi_{2}=0$ is analyzed in Sec. IV. Reflection of an em wave from a succession of impulsive waves follows in Sec. V and we complete the paper with our conclusion in Sec. VI.

\section{HORIZON FORMING EINSTEIN-SCALAR AND EM-SCALAR SOLUTIONS}

In order to obtain $\mathrm{CH}$ forming solutions with scalar fields we choose $\mathrm{CH}$ forming pure em and pure gravitational solutions known beforehand. For the pure gravitational solution we choose the solution found by Yurtsever [6] and independently by Ferrari and Ibanez [7]. An inter- esting property of this solution is that, its interaction region is isometric to the part of the interior region of the Schwarzschild black hole.

As for the pure em case we employ the well-known Bell-Szekeres solution [8]. The scalar field is added to both solutions by the $M$-shift method which we had explained elsewhere [9]. For completeness we summarize this method briefly as follows: The general metric for colliding plane waves is represented by [1]

$$
\begin{aligned}
d s^{2}= & 2 e^{-M} d u d v-e^{-U}\left\{\left[e^{V} d x^{2}+e^{-V} d y^{2}\right] \cosh W\right. \\
& -2 \sinh W d x d y\}
\end{aligned}
$$

in which the metric functions depend at most on the null coordinates $u$ and $v$. The basic field equations of colliding Einstein-Maxwell-scalar (CEMS) system can be generated from the following Lagrangian density (for parallel polarization, $W=0$ ),

$$
\begin{aligned}
L= & e^{-U}\left\{M_{u} U_{v}+M_{v} U_{u}+U_{u} U_{v}-V_{u} V_{v}-4 \phi_{u} \phi_{v}\right. \\
& \left.-2 e^{U+V} A_{u} A_{v}\right\} .
\end{aligned}
$$

Varying this Lagrangian yields the following CEMS field equations,

$$
\begin{gathered}
U_{u v}=U_{u} U_{v}, \\
2 M_{u v}=-U_{u} U_{v}+V_{u} V_{v}+4 \phi_{u} \phi_{v}, \\
2 V_{u v}=U_{v} V_{u}+U_{u} V_{v}-2\left(\bar{\Phi}_{0} \Phi_{2}+\bar{\Phi}_{2} \Phi_{0}\right), \\
2 \phi_{u v}=U_{v} \phi_{u}+U_{u} \phi_{v}, \\
2 A_{u v}=V_{v} A_{u}+V_{u} A_{v} .
\end{gathered}
$$

The remaining two equations

$2 U_{u u}-U_{u}^{2}+2 M_{u} U_{u}=V_{u}^{2}+4 \phi_{u}^{2}+4 \Phi_{2} \bar{\Phi}_{2}$,

$2 U_{v v}-U_{v}^{2}+2 M_{v} U_{v}=V_{v}^{2}+4 \phi_{v}^{2}+4 \Phi_{0} \bar{\Phi}_{0}$,

corresponding to $R_{u u}=-T_{u u}$ and $R_{v v}=-T_{v v}$ which do not follow from the variational principle are used to integrate for the metric function $M$. In these equations $\phi$ represents the massless scalar field, $\Phi_{0}$ and $\Phi_{2}$ are the Newman-Penrose spinors for em fields defined by

$$
\begin{aligned}
\Phi_{2} & =F_{\mu \nu} \bar{m}^{\mu} n^{\nu}=-\frac{e^{(U-V) / 2}}{\sqrt{2}} A_{u}, \\
\Phi_{0} & =F_{\mu \nu} l^{\mu} m^{\nu}=\frac{e^{(U-V) / 2}}{\sqrt{2}} A_{v}, \\
F_{\mu \nu} & =\partial_{\mu} A_{\nu}-\partial_{\nu} A_{\mu},
\end{aligned}
$$

where subscripts denote partial derivatives and overbar denotes the complex conjugate. The nonzero Weyl and Ricci scalars are defined by 


$$
\begin{aligned}
\Psi_{0} & =-C_{\mu \nu \rho \sigma} l^{\mu} m^{\nu} l^{\rho} m^{\sigma}, \\
\Psi_{2} & =-C_{\mu \nu \rho \sigma} l^{\mu} m^{\nu} \bar{m}^{\rho} n^{\sigma}, \\
\Psi_{4} & =-C_{\mu \nu \rho \sigma} n^{\mu} \bar{m}^{\nu} n^{\rho} \bar{m}^{\sigma}, \\
\Phi_{00} & =-\frac{1}{2} R_{\mu \nu} l^{\mu} l^{\nu}, \\
\Phi_{11} & =-\frac{1}{4} R_{\mu \nu}\left(l^{\mu} n^{\nu}+m^{\mu} \bar{m}^{\nu}\right), \\
\Phi_{22} & =-\frac{1}{2} R_{\mu \nu} n^{\mu} n^{\nu}, \\
\Phi_{02} & =-\frac{1}{2} R_{\mu \nu} m^{\mu} m^{\nu}, \\
\Lambda & =\frac{1}{24} R,
\end{aligned}
$$

where $C_{\mu \nu \rho \sigma}$ stands for the Weyl tensor, $R_{\mu \nu}$ the Ricci tensor and $R$ is the Ricci scalar. Weyl scalars have the following physical interpretation: The $\Psi_{0}$ and $\Psi_{4}$ terms represents the transverse gravitational wave components in the $n^{\mu}$ and $l^{\mu}$ direction, respectively. The $\Psi_{2}$ component is known as the "Coulomb-like" component that arises as a result of nonlinear interaction. The Ricci scalars $\Phi_{00}, \Phi_{11}$, $\Phi_{02}\left(=\bar{\Phi}_{20}\right), \Phi_{22}$ and $\Lambda$ on the other hand, corresponds to the matter fields. In terms of these Ricci scalars, the energy-stress tensor is given by

$$
\begin{aligned}
4 \pi T_{\mu \nu}= & \Phi_{00} n_{\mu} n_{\nu}+\Phi_{22} l_{\mu} l_{\nu}+\Phi_{02} \bar{m}_{\mu} \bar{m}_{\nu}+\Phi_{20} m_{\mu} m_{\nu} \\
& +\left(\Phi_{11}+3 \Lambda\right)\left(l_{\mu} n_{\nu}+n_{\mu} l_{\nu}\right) \\
& +\left(\Phi_{11}-3 \Lambda\right)\left(m_{\mu} \bar{m}_{\nu}+\bar{m}_{\mu} m_{\nu}\right) .
\end{aligned}
$$

We incorporate a scalar field $\phi(u, v)$ into this vacuum (or electrovacuum) metric through shifting the metric function $M$ in Eqs. (8), (i.e. the $M$-shift, for details see Ref. [9])

$$
M \rightarrow M+\Gamma
$$

where the scalar field is related to $\Gamma$ through the conditions

$$
U_{u} \Gamma_{u}=2 \phi_{u}^{2}, \quad U_{v} \Gamma_{v}=2 \phi_{v}^{2} .
$$

Stated otherwise, by shifting $M$ in accordance with (12) and through identifications (13) the shift function $\Gamma$ accounts for the scalar field in a consistent manner. The integrability condition imposes the massless scalar field equation as a constraint condition,

$$
2 \phi_{u v}-U_{u} \phi_{v}-U_{v} \phi_{u}=0 .
$$

The metric function $U$ is already known from the vacuum (electrovacuum) problem so that the crucial point is to choose a suitable scalar field that will yield a $\mathrm{CH}$ instead of a curvature singularity. To achieve this part of the problem it is often more convenient to pass to a prolate type of coordinates $(\tau, \sigma)$ instead of the null coordinates $(u, v)$. For the vacuum (electrovacuum) problem, the relation between the new coordinates $(\tau, \sigma)$ in terms of the null coordinates $(u, v)$ are defined by

$$
\tau=\sin (a u+b v), \quad \sigma=\sin (a u-b v),
$$

where $a, b$ are constants. In the prolate coordinates the massless scalar field Eq. (14) transforms into

$$
\left(\Delta \phi_{\tau}\right)_{\tau}-\left(\delta \phi_{\sigma}\right)_{\sigma}=0
$$

where $\Delta=1-\tau^{2}$ and $\delta=1-\sigma^{2}$. The exact solutions to above equation are already well known [1].

We observe that the choice

$$
\phi(\tau, \sigma)=a_{0} \tau \sigma \quad a_{0}=\text { constant, }
$$

solves Eq. (16) and more importantly it belongs to a class of $\mathrm{CH}$ forming solutions. Integrating Eqs. (13) for the metric function $\Gamma$ in terms of the $(\tau, \sigma)$ coordinates yields

$$
\Gamma=a_{0}^{2}\left(\tau^{2}+\sigma^{2} \Delta\right)
$$

This function is well-behaved and finite as the focusing hypersurface is approached. In the problem considered, the focusing hypersurface is given by

$$
e^{-U}=1-\sin ^{2} a u-\sin ^{2} b v=\sqrt{\Delta \delta}=0,
$$

and in terms of the prolate coordinates, focusing hypersurface is defined for $\tau=1$.

It should be noted that vanishing of the above equation at points $\sigma= \pm 1$ is not included in the interaction region. Those points represent the null boundaries separating the interaction region from the incoming regions.

The metric that describes the collision of plane impulsive gravitational wave accompanied with shock gravitational wave coupled with massless scalar field is obtained for linear polarization $(W=0)$ case as follows

$$
\begin{gathered}
e^{-U}=\sqrt{\Delta \delta}, \quad e^{-V}=\sqrt{\frac{\delta}{\Delta}}(1+\tau)^{2}, \\
e^{-M}=(1+\tau)^{2} e^{-\Gamma}
\end{gathered}
$$

The trace of the energy momentum tensor, $T(=R$, the scalar curvature) is obtained as

$$
T=R=\frac{4 a_{0}^{2} e^{\Gamma}}{(1+\tau)^{2}}\left(\tau^{2}-\sigma^{2}\right),
$$

while the Ricci and Weyl scalars are given by

$$
\begin{gathered}
\Phi_{00}^{(0)}=b^{2} \theta(v) a_{0}^{2} \sin ^{2}(2 b v) \\
\Phi_{22}^{(0)}=a^{2} \theta(u) a_{0}^{2} \sin ^{2}(2 a u) \\
\Phi_{11}^{(0)}=-\frac{1}{2} a b \theta(v) \theta(u) a_{0}^{2} \sin (2 a u) \sin (2 b v) \\
\Lambda^{(0)}=\frac{1}{6} a b \theta(v) \theta(u) a_{0}^{2} \sin (2 a u) \sin (2 b v) \\
\Psi_{2}^{(0)}=a b \theta(v) \theta(u)\left[\frac{1}{1+\tau}-a_{0}^{2} \sin (2 a u) \sin (2 b v)\right]
\end{gathered}
$$




$$
\begin{aligned}
\Psi_{4}^{(0)}= & a \delta(u)-\frac{3 a^{2} \theta(u)}{1+\tau}+a_{0}^{2} a^{2} \theta(u) \sin (2 a u) \\
& \times[2 \cos (a u-b v)-\sin (2 a u)], \\
\Psi_{0}^{(0)}= & b \delta(v)-\frac{3 b^{2} \theta(v)}{1+\tau}+a_{0}^{2} b^{2} \theta(v) \sin (2 b v) \\
& \times[2 \cos (a u-b v)-\sin (2 b v)],
\end{aligned}
$$

in which the scale invariant quantities are defined by $Z^{(0)}=$ $e^{-M} Z$. Note that the metric function $\Gamma$, the scalar $R$, and the Weyl and Ricci scalars all remain finite as the focusing hypersurface $\tau \rightarrow 1$ is approached. This indicates that curvature singularity does not develop on the focusing surface and hence, that surface is called a Cauchy-horizon surface.

Similarly, the metric that describes $\mathrm{CH}$ forming emscalar waves is

$$
e^{-U}=\sqrt{\Delta \delta}, \quad e^{-V}=\sqrt{\frac{\delta}{\Delta}}, \quad e^{-M}=e^{-\Gamma},
$$

with the $\phi$ and $\Gamma$ once more as in Eqs. (17) and (18), respectively. The scale invariant Weyl and Ricci scalars in the present case are

$$
\begin{gathered}
\Psi_{2}^{(0)}=-a_{0}^{2} a b \theta(u) \theta(v)(\sin 2 a u)(\sin 2 b v), \\
\Psi_{4}^{(0)}=a \delta(u) \theta(v) \tan (b v)+a_{0}^{2} a^{2} \theta(u) \theta(v)(\sin 2 a u) \\
\times(\sin 2 b v), \\
\Psi_{0}^{(0)}=\quad b \delta(v) \theta(u) \tan (a u)+a_{0}^{2} b^{2} \theta(u) \theta(v)(\sin 2 a u) \\
\times(\sin 2 b v), \\
\Phi_{00}^{(0)}=b^{2}\left[1+a_{0}^{2} \sin ^{2}(2 b v)\right] \theta(v), \\
\Phi_{22}^{(0)}=a^{2}\left[1+a_{0}^{2} \sin ^{2}(2 a u)\right] \theta(u), \\
\Phi_{11}^{(0)}=-\frac{1}{2} a_{0}^{2} a b \sin (2 a u) \sin (2 a v) \theta(u) \theta(v), \\
\Lambda^{(0)}=\frac{1}{6} a_{0}^{2} a b \theta(u) \theta(v) \sin (2 a u) \sin (2 a v),
\end{gathered}
$$

which are all well-behaved functions in the interaction region $(u>0, v>0)$. The distributional singularities are explicitly seen in (31) and (32) which lie on the light cone. The nonzero scalar curvature invariant is given by

$$
\begin{aligned}
I & =\frac{1}{16} R_{\mu \nu \rho \sigma} R^{\mu \nu \rho \sigma}=2\left(\Psi_{0} \Psi_{4}+3 \Psi_{2}^{2}\right) \\
& =8 a_{0}^{4} a^{2} b^{2} \theta(u) \theta(v) e^{2 \Gamma} \sin ^{2}(2 a u) \sin ^{2}(2 b v),
\end{aligned}
$$

which hosts no singularities.

\section{REFLECTION OF EM WAVES IN THE COLLIDING EINSTEIN-SCALAR AND EM-SCALAR WAVES}

In the previous section, we have demonstrated, how the $\mathrm{CH}$ forming colliding wave metrics can be constructed in the Einstein-scalar and Maxwell-scalar theory. In this section, we shall consider the collision of the plane wave packets that contain; plane impulsive gravitational waves accompanied with shock gravitational waves coupled with massless scalar fields in one of the incoming region II $(u>$ $0, v<0)$, while in the other incoming region III $(u<0$, $v>0$ ), we have plane em wave coupled with massless scalar fields (see Fig. 1).

\section{A. Region II: $u>0, v<0$}

This region contains an Einstein-scalar plane wave which is represented by the following metric

$$
\begin{aligned}
d s^{2}= & 2(1+\sin a u)^{2} e^{-\Gamma(u)} d u d v \\
& -e^{-U(u)}\left\{e^{V(u)} d x^{2}+e^{-V(u)} d y^{2}\right\},
\end{aligned}
$$

where

$$
\begin{gathered}
\Gamma(u)=a_{0}^{2} \sin ^{2} a u\left(1+\cos ^{2} a u\right), \quad e^{-U(u)}=\cos ^{2} a u, \\
e^{-V(u)}=(1+\sin a u)^{2},
\end{gathered}
$$

and the scalar field is

$$
\phi(u)=a_{0} \sin ^{2} a u .
$$

We note that the null coordinate $u$ is implied with a step function $u \rightarrow u \theta(u)$ in all these expressions to assure that for $u<0$ we have a flat space. This metric has the gravitational wave component

$$
\begin{aligned}
\Psi_{4}^{(0)}= & a \delta(u)-a^{2} \theta(u)\left[\frac{3}{1+\sin a u}-2 a_{0}^{2} \cos a u\right. \\
& \times \sin 2 a u(1-\sin a u)],
\end{aligned}
$$

which shows that it is a mixture of impulsive and shock waves.

\section{B. Region III: $u<0, v>0$}

This region contains an em-scalar plane wave which is given by the following metric,

$$
d s^{2}=2 e^{-\Gamma(v)} d u d v-\cos ^{2} b v\left(d x^{2}+d y^{2}\right)
$$

with

$$
\begin{aligned}
& e^{-U(v)}=\cos ^{2} b v, \quad \Gamma(v)=a_{0}^{2} \sin ^{2} b v\left(1+\cos ^{2} b v\right), \\
& \phi(v)=-a_{0} \sin ^{2} b v,
\end{aligned}
$$

and em potential $A_{\mu}=A \delta_{\mu}^{y}$ with

$$
A=\sqrt{2} \sin b v .
$$


The corresponding Maxwell field is then

$$
\Phi_{0}^{(0)}(v)=-b(\cos b v) e^{U / 2}=-b,
$$

where $\Phi_{0}^{(0)}=e^{-M / 2} \Phi_{0}$ (and similarly $\left.\Phi_{2}^{(0)}=e^{-M / 2} \Phi_{2}\right)$.

\section{Region IV: $u>0, v>0$}

In order to obtain a solution in region IV which represents the collision of these waves; we impose the same condition as Griffiths had introduced in Refs. [1-3]. This condition is to take the metric function $V=V(u)$. With this choice, the basic field Eqs. (3)-(9) of CEMS system simplifies to a great extend. The complete solution is given by

$$
\begin{aligned}
e^{-U} & =\cos ^{2} a u+\cos ^{2} b v-1, \\
e^{-V} & =(1+\sin a u)^{2}, \\
e^{-M} & =(\cos a u)(\cos b v) e^{-\Gamma-V+U / 2}, \\
\phi & =a_{0}\left(\sin ^{2} a u-\sin ^{2} b v\right), \\
\Gamma & =a_{0}^{2}\left(\sin ^{2} a u+\sin ^{2} b v\right)\left(\cos ^{2} a u+\cos ^{2} b v\right), \\
A_{y} & =\sqrt{2} \sin b v(1+\sin a u), \\
\Phi_{0}^{(0)} & =-b \theta(v)(\cos b v) e^{U / 2}, \\
\Phi_{2}^{(0)} & =\left(\frac{V_{u}}{2}\right)(\sin b v) e^{U / 2},
\end{aligned}
$$

in which the null coordinates are as usual with the step functions, $u=u \theta(u)$ and $v=v \theta(v)$.

The presence of $\Phi_{2}^{(0)}$ of the Maxwell field indicates that the em field reflects from the gravitational field. The nonzero Weyl components are

$$
\begin{aligned}
\Psi_{2}^{(0)}= & -a b \theta(u) \theta(v)(\sin 2 a u)(\sin 2 b v)\left(a_{0}^{2}+\frac{e^{2 U}}{4}\right), \\
\Psi_{4}^{(0)}= & a \delta(u)-a^{2} \theta(u)(1-\sin a u)\left\{\frac{1}{1+\sin a u}\right. \\
& \left.+e^{U} \sin a u-\frac{2}{3} a_{0}^{2} \sin a u[\cos 2 a u+\cos 2 b v]\right\},
\end{aligned}
$$

which indicate a curvature singularity on $e^{-U}=0$. The absence of the $\Psi_{0}$ component reveals that the gravitational field does not reflect from the em field. Our result verifies once more, in conform with the Mariot-Robinson theorem [1] that reflection of em waves from gravitational waves in a cosmology dominated by the particular scalar field considered above remains unchanged. This result could not be anticipated a priori, unless proved explicitly.

\section{A PARTICULAR SCALAR FIELD THAT MAKES $\Psi_{2}=0$}

The metric function $M$ is the one that determines the Weyl component $\Psi_{2}$ since

$$
\Psi_{2}^{(0)}=\frac{1}{2} M_{u v}
$$

Is it possible to choose a scalar field that will lead to a separable $M(u, v)$ of the form $M(u, v)=M_{1}(u)+M_{2}(v)$ ? This will enable us to set $\Psi_{2}=0$, leaving behind only $\Psi_{4}$ for the gravitational field. By inspecting (46) we see that the choice

$$
\phi(u, v)=\Gamma(u, v)=\frac{1}{2} U(u, v),
$$

does the trick and still it leads to an exact solution. In particular Eqs. (13) and (14) are automatically satisfied by virtue of the field equations. Thus, we can formulate our initial value problem of colliding Einstein-Maxwell-scalar fields as follows:

Region II:

$$
\begin{array}{ll}
\phi(u)=\Gamma(u)=\frac{1}{2} U(u), & e^{-U(u)}=\cos ^{2} a u, \\
e^{-V(u)}=(1+\sin a u)^{2}, & e^{-M}=(\cos a u) e^{-V(u)}
\end{array}
$$

Region III:

$$
\begin{gathered}
\phi(v)=\Gamma(v)=\frac{1}{2} U(v), \quad e^{-U(v)}=\cos ^{2} b v, \\
e^{-V}=1, \quad \Phi_{0}^{(0)}(v)=-b \theta(v), \\
A_{y}=\sqrt{2} \sin b v, \quad e^{-M}=\cos b v .
\end{gathered}
$$

These initial data imposed from right and left results in the following interaction region.

Region IV:

$$
\begin{aligned}
e^{-U} & =\cos ^{2} a u+\cos ^{2} b v-1,=\left(b 7^{V} \sin a u\right)^{2}, \\
e^{-M} & =(\cos a u)(\cos b v) e^{-V}, \\
\phi(u, v) & =\Gamma(u, v)=\frac{1}{2} U(u, v), \\
\Phi_{0}^{(0)} & =-b \theta(v)(\cos b v) e^{U / 2}, \\
\Phi_{2}^{(0)} & =\left(\frac{V_{u}}{2}\right)(\sin b v) e^{U / 2} .
\end{aligned}
$$

It is manifest now, by virtue of the chosen scalar field that we have

$$
\begin{aligned}
\Psi_{2}= & 0, \\
\Psi_{4}^{(0)}= & a \delta(u)-a^{2} \theta(u)(1-\sin a u) \\
& \times\left[\frac{3}{1+\sin a u}+2 e^{U} \sin a u\right] .
\end{aligned}
$$

We remark that this technique of making $\Psi_{2}=0$, by using a scalar field may have a larger scope within the context of colliding waves. It may not be possible however, to find a physical scalar field that satisfies the criteria. As an example we recall the Khan-Penrose (KP) metric of colliding impulsive gravitational waves [10]. Even when 
we complexify the scalar field we end up with

$$
\sqrt{2}\left|\phi_{u}\right|=\sqrt{\Gamma_{u} U_{u}}, \quad \sqrt{2}\left|\phi_{v}\right|=\sqrt{\Gamma_{v} U_{v}} .
$$

It can readily be seen from $\Gamma=-M_{\mathrm{KP}}$, that $\Gamma_{u} U_{u}<0$ $\left(\Gamma_{v} U_{v}<0\right)$ so that such a scalar field does not exist and we can not make $\Psi_{2}=0$. A similar calculation for the Yurtsever [6] solution reveals, however that such a scalar field does exist albeit it is tedious to be determined.

The fact that the role of a gravitational degree of freedom can be taken over by a scalar field in the problem of colliding waves is not new. We recall that by identifying the metric function $V$ by the scalar field $\phi$ the problem/ solution remains invariant [11]. This amounts to the fact that a linearly polarized gravitational wave can be imitated by a scalar field $\phi$ with $V=0$. We add that when the gravitational waves are cross polarized such an identification with either real or complex scalar field fails.

We wish to add that by shifting $v \theta(v) \rightarrow-v \theta(-v)$ the foregoing solution remains intact while the physical interpretation (see Fig. 2) changes drastically [12]: An incoming em field coupled with a scalar field characterized by the step function $\theta(-v)$ reflects from a curved region valid for $u>0, v<0$ and transforms into an outgoing gravitational field coupled with scalar wave. This scenario arises as a

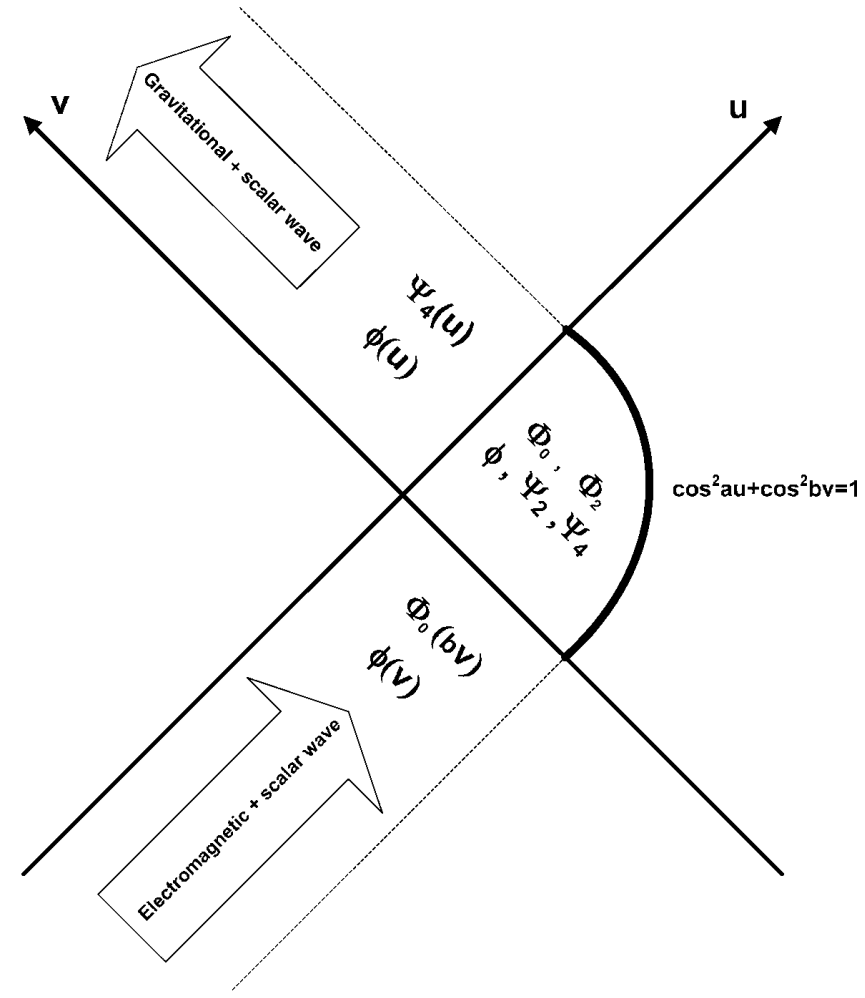

FIG. 2. The space-time diagram describes the reflection of an em + scalar wave wave packet from a curved space-time with a timelike singularity and transforming into an outgoing gravitational + scalar wave packet. This kind of interpretation is the result of applying $v \theta(v) \rightarrow-v \theta(-v)$ in Fig. 1 . result of the invariance of the field equations and their solution under $v \theta(v) \rightarrow-v \theta(-v)$. Setting the scalar field to zero is interpreted in anology as an em wave reflecting from the curved region implicated by the Griffiths's solution. We must admit that the physical interpretation of such a wave transmutation in general relativity remains still obscure.

\section{REFLECTION OF EM WAVE FROM A SUCCESSION OF GRAVITATIONAL IMPULSIVE WAVES}

The generic form of the metric in region II, for parallel polarization can be taken as

$$
d s^{2}=2 d u d v-(F d x)^{2}-(G d y)^{2},
$$

where $F$ and $G$ are only functions of $u$. For the single impulsive wave located at $u=0$ we have

$$
F=1+u \theta(u), \quad G=1-u \theta(u) .
$$

Now if we superimpose a second impulsive wave at the wave front $u=u_{1}>0$, our $F$ and $G$ functions become

$$
\begin{aligned}
& F=1+u \theta(u)-\frac{2}{1-u_{1}}\left(u-u_{1}\right) \theta\left(u-u_{1}\right), \\
& G=1-u \theta(u)+\frac{2}{1+u_{1}}\left(u-u_{1}\right) \theta\left(u-u_{1}\right),
\end{aligned}
$$

in which the coefficients are chosen deliberately such that

$$
e^{-U}=F G=1-u^{2} \theta(u),
$$

still holds. Similarly, for the 3-waves case we have

$$
\begin{aligned}
F= & 1+u \theta(u)-\frac{2}{1-u_{1}}\left(u-u_{1}\right) \theta\left(u-u_{1}\right) \\
& +\frac{2}{1-u_{1}} \frac{1+u_{1}}{1+u_{2}}\left(u-u_{2}\right) \theta\left(u-u_{2}\right), \\
G= & 1-u \theta(u)+\frac{2}{1+u_{1}}\left(u-u_{1}\right) \theta\left(u-u_{1}\right) \\
& -\frac{2}{1+u_{1}} \frac{1-u_{1}}{1-u_{2}}\left(u-u_{2}\right) \theta\left(u-u_{2}\right),
\end{aligned}
$$

which also satisfies (58) for $u_{2}>u_{1}>0$. It is not difficult to write a similar expression for an arbitrary number of successive waves [13]. The remaining metric function $V$ is given by

$$
e^{V}=\frac{F}{G}
$$

Since it is the expression $V_{u}$ that enters into the field equations we wish to give its form for different cases: 


$$
\begin{aligned}
\text { 2-waves: } V_{u} & =\frac{2}{1-u^{2}}\left[\theta(u)-2 \theta\left(u-u_{1}\right)\right], \\
\text { 3-waves: } V_{u} & =\frac{2}{1-u^{2}}\left[\theta(u)-2 \theta\left(u-u_{1}\right)+2 \theta\left(u-u_{2}\right)\right], \\
n+\text { 1-waves: } V_{u} & =\frac{2}{1-u^{2}}\left[\theta(u)+2 \sum_{i=1}^{n}(-1)^{i} \theta\left(u-u_{i}\right)\right] .
\end{aligned}
$$

The impulsive wave fronts $u_{i}$ must obviously satisfy $0 \leq u_{i}<1$ and we have the ordering relation $u_{i}<u_{j}$ for $i<j$. In between each of the successive waves our space-time is naturally flat. We note that in taking $\left(V_{u}\right)^{2}$, which appears in the field equations we adopt the standard properties of the step functions, such as

$$
\theta\left(u-u_{i}\right) \theta\left(u-u_{j}\right)=\theta\left(u-u_{j}\right),
$$

for $i<j$. As a result we obtain

$$
\left(V_{u}\right)^{2}=\frac{4 \theta(u)}{\left(1-u^{2}\right)^{2}},
$$

which implies that this term effectively is equivalent to a single wave located at $u=0$. In Fig. 3, we show the collision problem of an em wave with a train of 3-waves system. The Weyl components of the succesive waves can be found easily. We have for

$$
\begin{aligned}
\text { 2-waves: } \Psi_{4} & =-\delta(u)+\frac{2}{1-u_{1}^{2}} \delta\left(u-u_{1}\right), \\
\text { 3-waves: } \Psi_{4} & =-\delta(u)+\frac{2}{1-u_{1}^{2}} \delta\left(u-u_{1}\right)-\frac{2}{1-u_{2}^{2}} \delta\left(u-u_{2}\right), \\
n+1 \text {-waves: } \Psi_{4} & =-\delta(u)-2 \sum_{i=1}^{n} \frac{(-1)^{i}}{1-u_{i}^{2}} \delta\left(u-u_{i}\right) .
\end{aligned}
$$

For completeness we remind that for the present impulsive plane wave space-time the nonzero Riemann tensor components are related to $\Psi_{4}$ by

$$
R_{\text {uxux }}=-e^{V-U} \Psi_{4}, \quad R_{\text {uyuy }}=e^{-V-U} \Psi_{4} .
$$

For the 2-waves case, as an example, we have

$$
R_{\text {uxux }}=\delta(u)-2 \frac{1+u_{1}}{1-u_{1}} \delta\left(u-u_{1}\right)=-R_{\text {uyuy }} .
$$

It is manifest from these expressions that in between each pair of successive waves we have a flat space. We remind also that the case of shock gravitational waves is different. The problem of colliding em wave with a shock sandwich wave in which the incoming Weyl scalar is of the form

$$
\Psi_{4}=a^{2}\left[\theta(u)-\theta\left(u-u_{1}\right)\right],
$$

was considered before [14]. Clearly this case has a uniform curvature filling the sandwich and is entirely different from the present case. The solution of colliding em wave (with the same initial data as in the previous section and without the scalar field) is given by

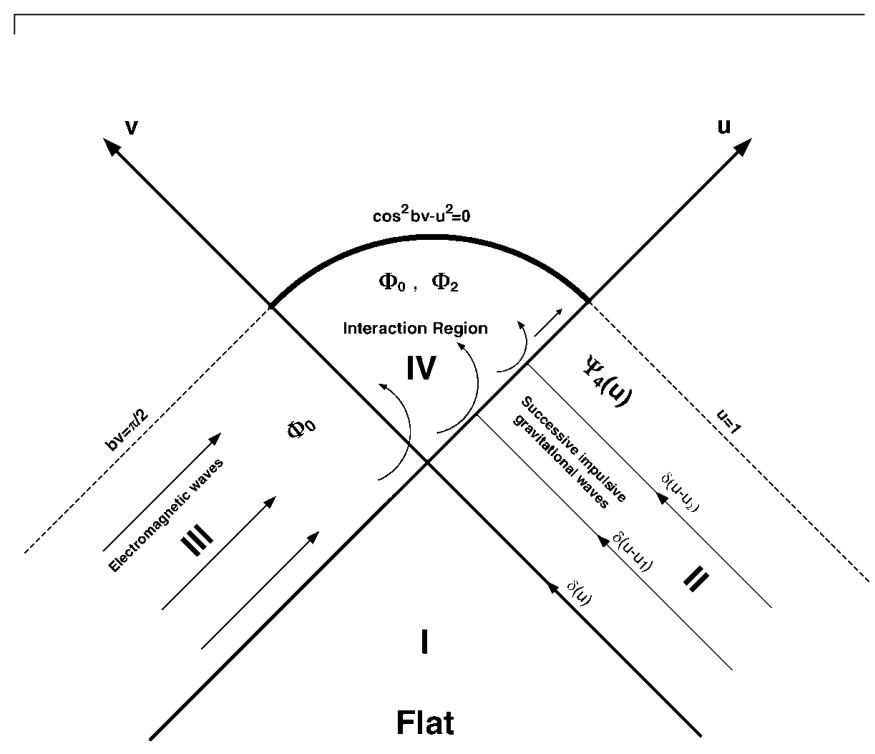

FIG. 3. The space-time diagram describes the reflection process of an em wave from a succession of three impulsive gravitational waves. The process results in a curvature singularity on the focusing surface $\cos ^{2} b v-u^{2}=0$. 


$$
\begin{aligned}
e^{-U} & =\cos ^{2} b v-u^{2}, \\
e^{V} & =\frac{F}{G}, \\
e^{-M} & =(\cos b v) \sqrt{1-u^{2}} e^{U / 2}, \\
A_{y} & =\sqrt{2}(\sin b v) e^{-V / 2}, \\
\Phi_{0}^{(0)} & =-b \theta(v)(\cos b v) e^{U / 2}, \\
\Phi_{2}^{(0)} & =\left(\frac{V_{u}}{2}\right)(\sin b v) e^{U / 2},
\end{aligned}
$$

where $F$ and $G$ are given in terms of the impulsive waves in succession. For 2-wave and 3-wave cases we have given them explicitly in (57) and (59). It is observed that although the Maxwell component $\Phi_{0}$ remains unaffected by the successive waves, $\Phi_{2}$ component changes sign each time when the em wave encounters a new wave front. This amounts to a phase change by $180^{\circ}$ of the reflected $\Phi_{2}$ component of the em wave. Overall effect is that if it crosses an even number of impulsive waves we have $\Phi_{2} \rightarrow$ $-\Phi_{2}$ while for an odd number of waves the sign remains unchanged. This sign change is physically significant since it shows itself in the em invariant $I=\frac{1}{4} F_{\mu \nu} F^{\mu \nu}=2 \Phi_{0} \Phi_{2}$.

To conclude this section we state that by the $M$-shift method we can easily extend the present consideration of successive impulsive waves to the case of EinsteinMaxwell-scalar case, which we shall not elaborate.

\section{CONCLUSION}

It is shown that reflection of em waves from gravitational waves with added scalar fields and with superposition of impulsive waves still satisfies the solution given long time ago by Griffiths. Solutions were also found in the past in which gravitational waves reflect from scalar waves while em waves do not reflect from scalar waves [15]. In particular we choose our initial data from the $\mathrm{CH}$ forming waves to see whether a nonsingular reflection process results. Our conclusion is that the mutual focusing is still quite strong to yield a curvature singularity. It remains open to find a $\mathrm{CH}$ forming reflection process yet. We can also exploit the presence of the scalar field by making a particular choice which sets $\Psi_{2}=0$, giving rise to a novel case without a Coulomb component in the interaction region. We have given also a counter example (the KhanPenrose case) which implies that this condition can not be generic.

The second part of the paper presents a multiple reflection process of an em wave from layers of successive impulsive gravitational waves. While $\Phi_{0}$ remains invariant, $\Phi_{2}$ is multiplied by $(-1)^{n+1}$, for $n \geq 1$, representing the number of superposed waves. This succesive sign flip in the Maxwell spinor is the precursor of a train of gravitational impulsive waves and may be searched for an indirect evidence of the latter. As a final remark we note that the reflection process is applicable only to a linearly polarized em wave. If cross-polarized em wave is considered, the condition $V=V(u)$ (and $W=W(u)$ ) remains no more feasible [16]. It remains to be seen whether it works in higher dimensional string theory where scalar field is replaced by dilaton and em field is replaced by the higher form fields. From physics standpoint it is an utmost important and fashionable problem nowadays to understand the classical scattering of wave packets in general relativity since black holes are believed to be produced in abundance in such processes [17]. While investing much effort on reflections from the horizons of black holes, it will not be adequate to ignore reflection processes of waves from other systems, as considered in this paper.

\section{ACKNOWLEDGMENTS}

One of us (O.G.) would like to thank to the Physics Department of the Bogazici University for their kind hospitality.
[1] J.B. Griffiths, Colliding Plane Waves in General Relativity (Oxford University Press, Oxford, 1991).

[2] J. B. Griffiths, Phys. Lett. A 54, 269 (1975).

[3] J. B. Griffiths, Ann. Phys. (N.Y.) 102, 388 (1976).

[4] C. Barrabes and P. A. Hogan, Singular Null Hypersurfaces in General Relativity (World Scientific, Singapore, 2004).

[5] G. Bressange, Classical Quantum Gravity 15, 225 (1998).

[6] U. Yurtsever, Phys. Rev. D 38, 1706 (1988).

[7] V. Ferrari and J. Ibanez, Gen. Relativ. Gravit. 19, 405 (1987).

[8] P. Bell and P. Szekeres, Gen. Relativ. Gravit. 5, 275 (1974).

[9] O. Gurtug, M. Halilsoy, and I. Sakalli, Gen. Relativ. Gravit. 35, 2159 (2003).
[10] K. A. Khan and R. Penrose, Nature (London) 229, 185 (1971).

[11] M. Halilsoy, Lett. Nuovo Cimento 44, 544 (1985).

[12] T. Dray and G. 't Hooft, Classical Quantum Gravity 3, 825 (1986).

[13] M. Halilsoy, Phys. Rev. D 42, 2922 (1990).

[14] A. Badawi and M. Halilsoy, Il Nuovo Cimento B 114, 21 (1999).

[15] W.Z. Chao, J. Phys. A 15, 2429 (1982).

[16] M. Halilsoy, Phys. Rev. D 37, 2121 (1988).

[17] S. B. Giddings and V.S. Rychkov, Phys. Rev. D 70, 104026 (2004). 